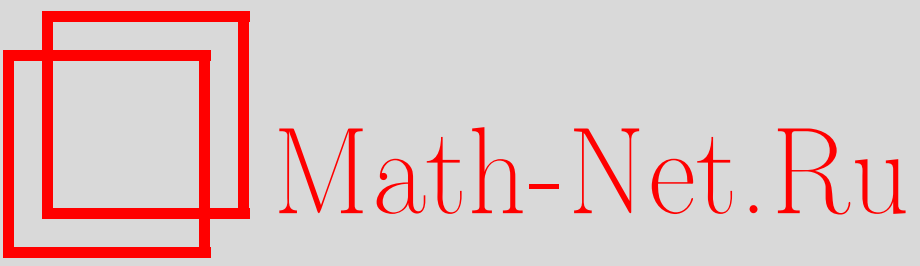

И. А. Андреева, А. Ф. Андреев, Качественное исследование в круге Пуанкаре одного семейства динамических систем, Итоги науки и техн. Сер. Соврем. мат. и ее прил. Темат. обз., 2020, том 185, 19-27

DOI: https://doi.org/10.36535/0233-6723-2020-185-19-27

Использование Общероссийского математического портала Math-Net.Ru подразумевает, что вы прочитали и согласны с пользовательским соглашением

http: //www.mathnet.ru/rus/agreement

Параметры загрузки:

IP : 54.224 .135 .184

26 апреля 2023 г., 10:01:22 


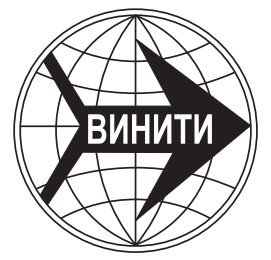

ИТОГИ НАУКИ И ТЕХНИКИ.

Современная математика и ее приложения.

Тематические обзоры.

Том 185 (2020). С. 19-27

DOI: $10.36535 / 0233-6723-2020-185-19-27$

УДК 517.938.25

\title{
КАЧЕСТВЕННОЕ ИССЛЕДОВАНИЕ В КРУГЕ ПУАНКАРЕ ОДНОГО СЕМЕЙСТВА ДИНАМИЧЕСКИХ СИСТЕМ
}

\author{
(ㄷ) 2020 г. $\quad$ И. А. АНДРЕЕВА, А. Ф. АНДРЕЕВ
}

\begin{abstract}
АннотАция. В статье рассматривается обширное семейство динамических систем, характерным признаком которых служит наличие у них полиномиальных правых частей, содержащих взаимно простые формы фазовых переменных системы. Одно из уравнений системы содержит полином третьей степени (форму кубическую), другое уравнение-квадратичную. Ставится задача построения в круге Пуанкаре всех возможных для систем данного семейства фазовых портретов и установления близких к коэффициентным критериев реализации каждого портрета. Задача решается с применением метода Пуанкаре последовательных отображений (центрального и ортогонального), а также целого ряда методов, специально разработанных авторами для целей данного исследования. Получены строгие результаты, как качественные, так и количественные. Построено свыше 250 топологически различных фазовых портретов систем семейства. Доказано отсутствие у систем данного семейства предельных циклов. Разработанные методы исследования могут быть полезны при дальнейшем изучении систем с полиномиальными правыми частями иного вида, как в чисто теоретическом, так и в прикладном аспектах.
\end{abstract}

Ключевъе слова: динамическая система, круг Пуанкаре, сфера Пуанкаре, траектория, фазовое пространство, фазовый портрет, сепаратриса, полиномиальная правая часть, особая точка, предельный цикл.

\section{QUALITATIVE RESEARCH IN THE POINCARÉ CIRCLE OF ONE FAMILY OF DYNAMICAL SYSTEMS}

\author{
(c) 2020 I. A. ANDREEVA, A. F. ANDREEV
}

\begin{abstract}
In this paper, we discuss an extensive family of dynamical systems whose characteristic feature is a polynomial right-hand side containing coprime forms of the phase variables of the system. One of the equations of the system contains a third-degree polynomial (cubic form), the other equation contains a quadratic form. We consider the problem of constructing all possible phase portraits in the Poincaré circle for systems from the family considered and establish criteria for the realization of each portrait that are close to coefficient criteria. This problem is solved by using the central and orthogonal Poincaré methods of sequential mappings and a number of other methods developed by the authors for the purposes of this study. We obtained rigorous qualitative and quantitative results. More than 250 topologically distinct phase portraits of various systems were constructed. The absence of limit cycles of systems of this family is proved. Methods developed can be useful for the further study of systems with polynomial right-hand sides of other forms.
\end{abstract}

Keywords and phrases: dynamical system, Poincaré circle, Poincaré sphere, trajectory, phase space, phase portrait, separatrix, polynomial right-hand side, singular point, limit cycle.

AMS Subject Classification: 34A34, 34C05, 34C07, 34C23 
1. Введение. Как показал еще Анри Пуанкаре, нормальная вещественная автономная дифференциальная система второго порядка с полиномиальными правыми частями принципиально допускает полное качественное исследование на расширенной вещественной плоскости (см., например, [16, с. 241-249]). С тех пор подобные исследования были проведены разными авторами для целого ряда семейств систем указанного вида, а именно: для квадратичных систем, для однородных кубических систем, для систем с ненулевыми линейными членами и с однородными нелинейными членами третьей, пятой либо седьмой степени в правых частях, для которых особая точка $O(0 ; 0)$ суть центр или фокус, и для некоторых прочих (см. $[4-6,8,9,15,21])$. В настоящей работе мы проводим исследование семейства кубических систем.

Изучается широкое семейство динамических систем с полиномиальными правыми частями, заданное на вещественной плоскости своих фазовых переменных $x, y$ :

$$
\frac{d x}{d t}=p_{0} x^{3}+p_{1} x^{2} y+p_{2} x y^{2}+p_{3} y^{3}=X(x, y), \quad \frac{d y}{d t}=a x^{2}+b x y+c y^{2}=Y(x, y),
$$

где $X(x, y), Y(x, y)$ представляют собой взаимно простые формы от $x$ и $y, X$ - кубическая, $Y$ квадратичная форма, $X(0,1)>0, Y(0,1)>0$. Рассмотрим задачу построить все топологически различные фазовые портреты систем (1) в круге Пуанкаре и определить близкие к коэффициентным критерии реализации каждого из таковых портретов.

Для решения поставленной задачи используется метод последовательных отображений А. Пуанкаре (см. $[1,2,16])$ :

(i) центрального отображения (из центра $(0,0,1)$ сферы $\left.\Sigma: x^{2}+y^{2}+z^{2}=1\right)$ плоскости $O x y$, пополненной бесконечно удаленной прямой (т.е. плоскости $\overline{\mathbb{R}}_{x, y}^{2}$ ) на сферу Пуанкаре $\Sigma$ (с отождествленными диаметрально противоположными точками);

(ii) ортогонального отображения нижней замкнутой половины сферы $\Sigma$ на круг Пуанкаре $\bar{\Omega}$ : $x^{2}+y^{2} \leqslant 1$ (с отождествленными диаметрально противоположными точками его границы $\Gamma$; см. [16, с. 241-249]).

В процессе этой работы применяются первое и второе преобразования Пуанкаре. Исследование проводится в несколько этапов.

2. Исследование особой точки $O(0 ; 0)$ систем (1). Введем следующие понятия.

Символом $\phi(t, p)$, где $p=(x, y)$ - фиксированная точка, будем обозначать решение (движение) системы $(1)$ с начальными данными $(0, p)$.

Через $L_{p}$ обозначим траекторию движения $\phi(t, p), t \in I_{\max }$, а через $L_{p}^{+}$и $L_{p}^{-}-$ее положительную и отрицательную полутраектории соответственно.

$O$-Кривая системы - это ее полутраектория $L_{p}^{s},(p \neq 0, s \in\{+,-\})$, примыкающая к точке $O$ при $s t \rightarrow+\infty$.

$O^{+}$-Кривая (соответственно, $O^{-}$-кривая) системы - это ее $O$-кривая $L_{p}^{+}$(соответственно, $L_{p}^{+}$); $O_{+}$-кривая (соответственно, $O_{-}$-кривая) системы - это ее $O$-кривая, примыкающая к $O$ из области $x>0$ (соответственно, $x<0)$.

$T O$-Кривая системы - это ее $O$-кривая, которая, будучи дополнена точкой $O$, касается в ней некоторого луча.

Узловой пучок $N O$-кривых системы - это открытое непрерывное семейство ее $T O$-кривых $L_{p}^{s}$, где индекс $s$ фиксирован, $p \in \Lambda, \Lambda$ - простая открытая дуга, причем $L_{p}^{s} \cap \Lambda=p$.

Седловой пучок $S O$-кривых системы, сепаратриса точки $O$ - фиксированная $T O$-кривая, которая не входит в состав какого-либо пучка $N O$-кривых системы.

Символами $E, H, P$ будем обозначать эллиптический, гиперболический и параболический $O$ секторы системы соответственно (см. $[1,2,4-6,8,9,16])$.

Топологическим типом (Т-типом) особой точки $O$ системы будем называть слово $A_{O}$ из букв $N, S$ (слово $B_{O}$ из букв $E, H, P$ ), фиксирующее круговой порядок следования пучков $N, S$ ее $O$ кривых (ее $O$-секторов $E, H, P$ ) при обходе точки $O$ в положительном направлении, т.е. против хода часовой стрелки, начиная с некоторого из них.

Кроме того, будем использовать обозначения

$$
P(u)=X(1, u)=p_{0}+p_{1} u+p_{2} u^{2}+p_{3} u^{3}, \quad Q(u)=Y(1, u)=a+b u+c u^{2} .
$$


Замечание 1. Для любой системы (1)

(i) Т-тип особой точки $O$ в виде $B_{O}$ легко строится по ее Т-типу вида $A_{O}$ (см. $[4, \S 1]$ ) и наоборот (мы будем пользоваться обоими типами);

(ii) вещественные корни полинома $P(u)$ (полинома $Q(u)$ ) суть угловые коэффициенты изоклин бесконечности (изоклин нуля);

(iii) при выписывании вещественных корней ее полиномов $P(u), Q(u)$, раздельно или всех вместе, мы всегда нумеруем корни каждого из них в порядке возрастания.

Подробный анализ возможных вариантов топологического типа точки $O(0 ; 0)$ проведен в $[8,9$, $12,14,20]$. Доказано, что системы $(1)$ не имеют на плоскости $\mathbb{R}_{x ; y}^{2}$ предельных циклов. Действительно, такой цикл должен был бы окружать особую точку $O(0 ; 0)$ системы, и индекс Пуанкаре последней $I(O)$ должен был бы равняться единице (см. $[8,16,20])$. Однако формула Бендиксона для индекса изолированной особой точки гладкой динамической системы, имеющая вид $I(O)=1+(e-h) / 2$, где $e(h)$ - число эллиптических (гиперболических) $O$-секторов системы (см. $[9$, с. 559]) в совокупности с теоремой, доказанной нами в $[8,9]$, позволяют сделать вывод, что для особой точки $O(0 ; 0)$ любой системы $(1)$ индекс Пуанкаре равен нулю, $I(O)=0$.

Следствие 1. Для особой точки $O(0 ; 0)$ системы (1) возможсны одиннадиать различных Tтипов, причем во всех случалх указанная особая точка $O(0 ; 0)$ имеет не более четырех сепаратрис (т.е. 2, 3 или 4).

3. Бесконечно удаленные особые точки систем (1). В данном разделе при помощи метода преобразований Пуанкаре (см., например, [16, с. 241-249], [17,23]) мы анализируем поведение траекторий систем (1) в окрестности бесконечности. Первое преобразование Пуанкаре $x=1 / z$, $y=u / z,(u=y / x, z=1 / x)$ преобразует систему (1) в систему, которая в координатах Пуанкаре $u, z$ после замены времени $d t=-z^{2} d \tau$ принимает вид

$$
\frac{d u}{d \tau}=P(u) u-Q(u) z, \quad \frac{d z}{d \tau}=P(u) z
$$

где $P(u)=X(1, u)$ и $Q(u)=Y(1, u)$ - взаимно простые полиномы. Эта система определена на всей сфере $\Sigma$, включая ее экватор, и на всей $(u, z)$-плоскости $\alpha^{*}$, касающейся сферы $\Sigma$ в точке $C=(1,0,0)$. На плоскости $\overline{\mathbb{R}}_{u, z}^{2}$ мы и будем ее изучать, а полученные результаты проектировать на круг $\bar{\Omega}$, последовательно проектируя сначала плоскость $\mathbb{R}_{u, z}^{2}$ на сферу $\Sigma$ из ее центра, а затем нижнюю ее полусферу $\bar{H}$ на круг Пуанкаре $\bar{\Omega}$, т.е. на замкнутый единичный круг плоскости $\mathbb{R}_{x, y}^{2}$ с помощью ортогональной к нему проекции.

Для рассматриваемой здесь системы ось $z=0$ инвариантна (состоит из траекторий системы); на ней лежат ее особые точки $O_{i}\left(u_{i}, 0\right), i=\overline{0, m}$, где $u_{i}, i=\overline{1, m}$, - все вещественные корни полинома $P(u)$, а $u_{0}=0$; при этом может существовать такое значение $i_{0}, i=\overline{0, m}$, что $u_{i_{0}}=0$. Выявленные здесь точки будем называть бесконечно удаленными особыми точками (БО-точками) первого типа системы (1).

Второе преобразование Пуанкаре $x=v / z, y=1 / z,(v=x / y, z=1 / y)$ также взаимно однозначно отображает плоскость $\mathbb{R}_{x, y}^{2}$ на сферу $\Sigma$ с отождествленными диаметрально противоположными точками, рассматриваемую без ее экватора, а любую систему (1) преобразует в систему, которая в координатах $\tau, v, z$ имеет вид

$$
\frac{d v}{d \tau}=-X(v, 1)+Y(v, 1) v z, \quad \frac{d z}{d \tau}=Y(v, 1) z^{2} .
$$

Система, полученная вторым преобразованием Пуанкаре, определена на всей сфере Пуанкаре $\Sigma$ и на всей $(v, z)$-плоскости, касающейся сферы Пуанкаре в точке $D=(0,1,0)$ (см. [16, рис. 135]). Множество $z=0$ для нее инвариантно; на нем лежат особые точки вида $\left(v_{0}, 0\right)$, где $v_{0}$-любой вещественный корень полинома

$$
X(v, 1)=p_{3}+p_{2} v+p_{1} v^{2}+p_{0} v^{3} .
$$

Их естественно было бы назвать бесконечно удаленными особыми точками (БО-точками) второго типа системы $(1)$, но любая из этих точек, для которой $v_{0} \neq 0$, очевидно, совпадает с одной 
из точек первого типа, а именно, с точкой $\left(1 / v_{0}, 0\right)$, а число $v_{0}=0$ не является корнем полинома $X(x, 1)$, ибо $X(0,1)=p_{3} \neq 0$ для системы (1). Таким образом, справедливо следующее утверждение.

Следствие 2. Бесконечно удаленными особыми точками любой системы (1) являются лишь бесконечно удаленные особые точки первого типа.

При ортогональном проектировании нижней полусферы $H$ сферы $\Sigma$ на плоскость $O x y$ ее открытая часть $H$ взаимно однозначно отображается на открытый круг Пуанкаре, а ее граница $E$ (экватор сферы $\Sigma$ ) - на окружность $\Gamma$ - границу круга Пуанкаре. Следовательно, имеют место следующие факты:

(i) собственные траектории любой системы (1) (включая ее особую точку $O(0 ; 0)$ ) отображаются в круг Пуанкаре $\Omega$, заполняя его;

(ii) бесконечно удаленные ее траектории (включая БО-точки) отображаются на граничную окружность $Г$ круга $\Omega$, заполняя ее.

Следуя Пуанкаре, мы называем траектории первого из этих типов траекториями системы (1) в $\Omega$, а вторые - траекториями на Г).

Как следует из предыдущего, любой БО-точке $O_{i}\left(u_{i}, 0\right)$ системы $(1), i=\overline{1, m}$, на $\Gamma$ соответствуют две диаметрально противоположные точки $O_{i}^{+}\left(u_{i}, 0\right)$ и $O_{i}^{-}\left(u_{i}, 0\right)$, принадлежащие соответственно $\Gamma^{+}=\Gamma \cap\{x>0\}$ и $\Gamma^{-}=\Gamma \cap\{x<0\}$.

Следствие 3. Бесконечно удаленные особые точки $O_{0}^{ \pm}$любой системы (1) не имеют сепаратрис.

4. (m,n)-Семейства систем (1). Фазовый портрет в круге Пуанкаре для любой системы (1) последовательно определяют в основном следующие факторы:

(1) числовая пара $(m, n)$, где $m=3,2,1(n=2,1,0)$ - число изоклин бесконечности (изоклин нуля) системы;

(2) порядок следования этих $m+n$ ее изоклин при полуобходе точки $O(0 ; 0)$ в области $x>0$ в направлении возрастания $u(=y / x)$;

(3) расположение ее изоклин бесконечности относительно оси $y=0$.

В переводе на аналитический язык (т.е. в терминах вещественных корней полиномов $P(u), Q(u)$ системы (1)) указанные факторы принимают следующий вид:

(1) числовая пара $(m, n)$, где $m$ и $n$ - числа вещественных корней полиномов $P(u)$ и $Q(u)$ соответственно;

(2) порядок следования этих $m+n$ корней $P(u), Q(u)$, упорядоченных по возрастанию;

(3) расположение последовательности корней $P(u)$ на оси $u$ относительно точки $u=0$.

Определение 1. Пусть $(m, n)$ - фиксированная пара чисел. Совокупность всех систем (1), для каждой из которых полином $P(u)$ имеет $m$ вещественных корней, а полином $Q(u)-n$ вещественных корней, будем называть $(m, n)$-семейством систем $(1)$, а каждую его систему - $(m, n)$ системой. Из данного определения следует, что исходное семейство систем (1) состоит из следующих $(m, n)$-семейств этих систем: $(3,2),(2,2),(3,1),(2,1),(3,0),(2,0),(1,2),(1,1),(1,0)$.

Определение 2. Пусть $(m, n)$ - фиксированное семейство систем (1) из приведенного выше списка. Последовательность всех $m+n$ вещественных корней полиномов $P(u), Q(u)$ произвольной системы семейства, упорядоченных по возрастанию, будем обозначать символом (словом) ПКРQ, а ее возрастающие подпоследовательности $m$ корней полинома $P(u)$ и $n$ корней полинома $Q(u)-$ словами ПКР и ПКQ.

Примеры ПКРQ систем $(3,2)$-семейства:

(i) $u_{1}, u_{2}, u_{3}, q_{1}, q_{2}$, (здесь $u_{1}<u_{2}<u_{3}<q_{1}<q_{2}$ ),

(ii) $u_{1}, q_{1}, u_{2}, q_{2}, u_{3}$, (здесь $u_{1}<q_{1}<u_{2}<q_{2}<u_{3}$ ).

В каждом из этих примеров ПКР имеет вид $u_{1}, u_{2}, u_{3}$, а ПКQ - вид $q_{1}, q_{2}$. 
Лемма 1. Для систем любого $(m, n)$-семейства систем (1) существует $\rho=(m+n) ! / m ! n$ ! различных ПКРQ.

С учетом упорядоченности в любой ПКРQ корней каждого из полиномов $P(u), Q(u)$ справедливость утверждения леммы очевидна.

Присвоим каждой из различных ПКРQ систем $(m, n)$-семейства определенный номер $r$ от 1 до $\rho$ и обозначим ее символом $(\text { ПКРQ })_{r}$, а ее подпоследовательности ПКР и ПКQ - символами $(\Pi K \mathrm{P})_{r}$ и $(\text { ПКQ })_{r}$. Решать нашу основную задачу естественно отдельно для каждого из $(m, n)$ семейств, изучая их поочередно по единой программе, изложенной в п. 5.1.

5. Методика исследования систем (1). Введем понятие топодинамического типа (ТД-тип) особой точки для конечной особой точки $O(0 ; 0)$ системы. А-схема точки $O$, т.е. слово $A_{0}$, определяющее Т-тип точки $O$, модифицируется следующим образом: каждая из букв $N, S$ в его составе снабжается правым верхним индексом + или - в зависимости от того, состоит ли соответствующий ей пучок $O$-кривых из $O^{+}$-кривых или из $O^{-}$-кривых. Получающееся при этом слово $A_{0}$ трактуется как слово, определяющее топодинамический тип точки $O$, и называется ТД-типом этой точки, а каждый из его символов $N^{+}, N^{-}, S^{+}, S^{-}$- ТД-типом соответствующего ему пучка $O$-кривых.

Для любой бесконечно удаленной точки (БО-точки) $O_{i}\left(u_{i}, 0\right), i=\overline{0, m}$, понятие топодинамического типа вводится аналогично. Слова $A_{i}^{( \pm)}$, определяющие Т-тип точки $O_{i}$, модифицируются следующим образом: каждая из букв $N, S$ в них снабжается правым верхним индексом + или - в зависимости от того, с возрастанием или с убыванием $t$ примыкают $O_{i}$-кривые соответствующего ей пучка к точке $O_{i}$. Получающиеся при этом слова $A_{i}^{( \pm)}$определяют ТД-тип точки $O_{i}$, а слово $A_{i}^{+}\left(A_{i}^{-}\right)$- ТД-тип точки $O_{i}^{+}\left(O_{i}^{-}\right)$.

Бездорожной картой (БД-карта) системы будем называть графическую схему, которая строится следующим образом.

1. В круге $\bar{\Omega}$ изображается поле направлений системы: отмечаются все ее особые точки, проводятся ее изоклины нуля и бесконечности, указываются стрелками направления векторного поля $(\dot{x}(t), \dot{y}(t))$ в областях между этими изоклинами и на полуосях $y=0,|x|>0$, а также поля $\dot{u}(t)$ на границе Г круга $\bar{\Omega}$ (между БО-точками).

2. Возле каждой особой точки изображаются примыкающие к ней пучки $N, S$ полутраекторий системы с учетом их ТД-типов: каждый пучок $S^{+}\left(S^{-}\right)$, являющийся сепаратрисой этой точки, изображается одной дугой со стрелкой, обращенной к точке (от точки), каждый пучок $N^{+}$ $\left(N^{-}\right)$- двумя-тремя такими дугами. Эти дуги располагаются с той стороны от проходящей через эту точку изоклины нуля или бесконечности, в которой стрелки на них согласуются с таковыми на векторах поля в $\Omega$ и на $\Gamma$.

По БД-карте системы будем изучать глобальное продолжение в круге $\Omega$ каждой из ее сепаратрис следующим образом. Фиксируем произвольную особую точку системы в круге $\bar{\Omega}$ и произвольную $\alpha(\omega)$-сепаратрису $S^{-}\left(S^{+}\right)$этой точки (она прочерчена на БД-карте лишь в малой окрестности последней), после чего построим на БД-карте системы всю эту сепаратрису, вплоть до ее $\omega(\alpha)$-предельных множеств, руководствуясь правилом, которое в автодорожных терминах формулируется так: начав движение системы от выбранной особой точки по ее $\alpha$-сепаратрисе $S^{-}$ и попав по выходе из малой окрестности этой точки на «бездорожье» (где «дороги», т.е. сепаратрисы системы, мы и должны прочертить), движемся, воспринимая указатели векторного поля системы как предписывающие знаки, а символы ТД-типов пучков $N^{+}, S^{+}$особых точек - как знаки разрешенных парковок (начав движение от выбранной особой точки по ее $\omega$-сепаратрисе $S^{+}$, по выходе на «бездорожье» движемся, воспринимая те же указатели и символы в противоположном смысле). Траектория этого движения от старта до парковки и определяет возможное глобальное продолжение данной сепаратрисы.

Как мы увидим далее, для одних сепаратрис систем (1) глобальное продолжение определяется однозначно, для других существуют его варианты. В связи с этим примем следующее определение. 
Определение 3. Если глобальное продолжение сепаратрисы системы (1) в круге $\bar{\Omega}$ однозначно, будем называть эту сепаратрису обыкновенной, а в противном случае - особой.

Максимальной простой инвариантной ячейкой (МП-ячейкой) системы будем называть инвариантную для системы область круга $\bar{\Omega}$, для которой фазовый поток системы имеет один точечный источник и один точечный сток и которая не является собственной подобластью другой такой области. Как мы увидим далее, для каждой системы (1) ее сепаратрисы, будучи максимально продолжены по $t$ в круге $\bar{\Omega}$, разбивают этот круг на МП-ячейки $\bar{\Omega}_{i}, i=\overline{1, v}$, где $v$-число всех различных сепаратрис системы.

Двойную замену $(t, y) \rightarrow(-t,-y)$ будем называть ДЗ-преобразованием системы. Такая замена преобразует ее в систему, строго говоря, иную: для новой системы знаки и нумерации корней многочленов $P(u), Q(u)$, а также направление движения по траекториям с возрастанием $t$ изменяются на противоположные в сравнении с характерными для исходной системы.

Две различные системы будем называть взаимно обратными (относительно двойной замены), если ДЗ-преобразование трансформирует одну из них в другую, и независимыми (от ДЗ-преобразования) в противном случае.

5.1. Программа исследования систем (1). Нашу основную задачу естественно решать отдельно для каждого из $(m, n)$-семейств, изучая их поочередно по следующей общей программе.

1. Фиксируем очередное $(m, n)$-семейство. Под таковым мы понимаем подсемейство систем $(1)$ первого иерархического уровня, а именно подсемейство, правые части систем которого разлагаются на $m$ (для кубической формы) и $n$ (для квадратичной формы) различных множителей, т.е. их специальные полиномы $P(u), Q(u)$ имеют $m$ и $n$ несовпадающих между собою различных корней соответственно.

Описываем общие свойства систем этого семейства: указываем вид систем, вид их полиномов $P(u), Q(u)$, перечисляем их особые точки в круге Пуанкаре $\bar{\Omega}$, последовательно разбиваем его на подсемейства дальнейших иерархических уровней.

2. Находим топодинамические типы (ТД-типы) особых точек его систем.

3. Изучаем поведение в круге Пуанкаре сепаратрис его систем.

4. Строим все различные фазовые портреты его систем.

5.2. Метод построения фазовых портретов систем (1). Для построения фазовых портретов систем (1) используем описательный метод: для каждого фазового портрета строим таблицу, которая содержит перечень его МП-ячеек $\bar{\Omega}_{i}, i=\overline{1, v}$, с указанием для каждой из них ее границы, а также источника и стока ее фазового потока.

Границу каждой ячейки описываем словом из обозначений ее граничных сепаратрис, перечисляемых в порядке их следования при обходе ячейки по ее границе в положительном направлении (т.е. против хода часовой стрелки). Часть границы МП-ячейки, лежащую на $Г$, опускаем. Эту таблицу мы называем описательным фазовым портретом соответствующих систем и присваиваем ей номер k.r, k.r.s или k.r.s.l, где $k$ - номер $(m, n)$-семейства систем (1) по классификационному списку (см. $[7-9,11,12,14,19]), r, s, l$ - индексы его последовательных подсемейств, результатом поочередного изучения которых является эта таблица.

Для любой системы (1) ее фазовый портрет и описательный фазовый портрет дают одну и ту же информацию о поведении ее траекторий в круге Пуанкаре: первый - графически (рисунком), второй - описательно (таблицей). Мы строим для системы описательный фазовый портрет. Это позволяет сократить объем работы: часть описательного фазового портрета мы выписываем явно, остальные получаем экономным методом деформации базовой таблицы (см. ниже). Впрочем, если один из портретов - фазовый или описательный фазовый - системы получен, построить по нему второй - минутное дело (см. [4-6, 8, 9, 12-14,20,22]).

5.3. Метод деформации базовой таблицы. Метод деформации базовой таблицы состоит в следующем. Все не выписанные таблицы описательного фазового портрета $(m, n)_{r}$ и $(m, n)_{r ; s^{-}}$систем 
заменяем одной таблицей из трех-четырех колонок, построчная структура которой такова: в колонке 1 стоит номер очередной искомой таблицы описательного фазового портрета (ИТ), в колонке 2 - номер базовой таблицы описательного фазового портрета (БТ), т.е. некоторого известного описательного фазового портрета (выписанного явно или полученного в составляемой таблице), в колонке 3 указываются деформации базовой таблицы, превращающие ее в искомую таблицу.

Примеры деформаций базовой таблицы: удаление из базовой таблицы какой-либо строки, замена в ней одной-двух строк новой строкой, удаление из нее одних символов, замена или перестановка других. Если удаляемая из базовой таблицы строка не является последней, то в колонке 4 указывается необходимая перенумерация ее строк (ПСБТ).

Изучение $(m, n)$-семейств по программе п. 5.1 начинаем с первого из этих семейств, т.е. с $(3,2)-$ семейства.

6. Исследование $(3,2)$-систем. На первом этапе работы поставленная общая задача решается для тех систем (1), разложения форм $X(x, y)$ и $Y(x, y)$ которых на вещественные формы низших степеней содержат соответственно 3 и 2 множителя, т.е. $(3,2)$-систем:

$$
X(x, y)=p_{3}\left(y-u_{1} x\right)\left(y-u_{2} x\right)\left(y-u_{3} x\right), \quad Y(x, y)=c\left(y-q_{1} x\right)\left(y-q_{2} x\right),
$$

где $p_{3}>0, c>0, u_{1}<u_{2}<u_{3}, q_{1}<q_{2}, u_{i} \neq q_{j}$ при любых $i$ и $j$.

Для произвольной $(3,2)$-системы вводим следующие основные понятия. $P(u), Q(u)-$ ее полиномы $P, Q$ :

$$
P(u)=X(1, u) \equiv p_{3}\left(u-u_{1}\right)\left(u-u_{2}\right)\left(u-u_{3}\right), \quad Q(u)=Y(1, u) \equiv c\left(u-q_{1}\right)\left(u-q_{2}\right),
$$

ПКР (соответственно, ПКQ) - возрастающая последовательность всех вещественных корней ее полинома $P(u)$ (соответственно, $Q(u)$ ), ПKPQ - возрастающая последовательность всех вещественных корней обоих ее полиномов $P(u), Q(u)$.

Легко видеть, что для произвольной $(3,2)$-системы возможны 10 различных ПКРQ, поскольку $5 ! /(3 ! 2 !)=10$. ДЗ-преобразование $(3,2)$-систем позволяет определить, что шесть из них независимы попарно, в то время как у каждой из оставшихся четырех имеется взаимно обратная ей среди первых шести.

Каждой из различных ПКРQ $(3,2)$-системы присваиваем конкретный номер $r \in\{1, \ldots, 10\}$ так, чтобы $(\text { ПКРQ })_{r}$ с номерами $r=\overline{1,6}$ оказались попарно независимыми, а с номерами $r=\overline{7,10}-$ взаимно обратными системам с номерами $r=\overline{1,4}$ соответственно.

Вводится понятие $(3,2)_{r}$-семейство систем $(1)$ - совокупность всех систем $(3,2)$-семейства, для каждой из которых ПKРQ $=(\Pi \mathrm{KPQ})_{r}$.

Затем поочередно по единой схеме изучаются $(3,2)_{r}$-семейства систем $(1), r=\overline{1,6}$. Результаты для $(3,2)_{r}$-семейств, $r=\overline{7,10}$, получаются затем из результатов, полученных для $(3,2)_{r}$-семейств, $r=\overline{1,4}$, ДЗ-преобразованием систем последних.

Строятся фазовые портреты систем $(3,2)_{r}$-семейства как в графической форме (в виде рисунков), так и в описательной форме (в виде таблиц). Указываются критерии реализации каждого из них.

Итог первого этапа работы таков: для систем $(3,2)_{1}$-семейства возможны 25 различных фазовых портретов, для систем $(3,2)_{2}$ и $(3,2)_{3}$-семейств - по 9 фазовых портретов, для $(3,2)_{4}$ и $(3,2)_{5}$ семейств - по 7 , для систем $(3,2){ }_{6}$-семейства - 36, для всех $(3,2)$-систем - 93.

Это число как результат может производить впечатление немалого, но при его оценивании следует принять во внимание, что в каждом $(3,2)_{r}$-семействе число систем (1) несчетно (см. [3, $8,12,14])$.

7. Изучение $(2,2)$-семейства систем (1). Это семейство есть совокупность всех систем (1) вида

$$
\frac{d x}{d t}=p_{3}\left(y-u_{1} x\right)^{k_{1}}\left(y-u_{2} x\right)^{k_{2}}=X(x, y), \quad \frac{d y}{d t}=c\left(y-q_{1} x\right)\left(y-q_{2} x\right)=Y(x, y),
$$

где $p_{3}, c, u_{1}, u_{2}, q_{1}, q_{2}$ вещественны, $p_{3}>0, c>0, u_{1}<u_{2}, q_{1}<q_{2}, u_{i} \neq q_{j}$ для любых $i, j=1,2$, $k_{1}, k_{2}=1,2, k_{1}+k_{2}=3$. 
Естественно различать два класса таких систем: системы с $k_{1}=1$ и $k_{2}=2$ образуют класс $A$, в то время как системы с $k_{1}=2$ и $k_{2}=1-$ класс $B$. Для систем, относящихся к классу $A$, получены 45 топологически различных фазовых портрета, для систем же, принадлежащих к классу $B$, таковых насчитывается 52 .

Подробности и итоги исследования этого и прочих подсемейств, строгие доказательства теорем, а также тонкие детали, наряду с самими фазовыми портретами, читатель найдет в монографии [8] и статьях $[3-7,9-15,18-22]$.

\section{СПИСОК ЛИТЕРАТУРЫ}

1. Андреев А. Ф. Особые точки дифференциальных уравнений. - Минск, 1979.

2. Андреев $A . \Phi$. Введение в локальную качественную теорию дифференциальных уравнений. - СПб., 2003.

3. Андреев А. Ф., Андреева И. А. Фазовые потоки одного семейства кубических систем в круге Пуанкаpe, I// Диффер. уравн. процессы управл. - 2007. - 4. - С. 17-26.

4. Андреев А. Ф., Андреева И. А. Фазовые потоки одного семейства кубических систем в круге Пуанкаpe, II// Диффер. уравн. процессы управл. - 2008. - 1. - С. 1-13.

5. Андреев А. Ф., Андреева И. А. Фазовые потоки одного семейства кубических систем в круге Пуанкаpe, III// Диффер. уравн. процессы управл. - 2008. - 3. - С. 39-54.

6. Андреев А. Ф., Андреева И. А. Фазовые потоки одного семейства кубических систем в круге Пуанкаpe, $\mathrm{IV}_{1} / /$ Диффер. уравн. процессы управл. - 2009. - 4. - С. 181-213.

7. Андреева И. А. Об одном специальном семействе кубических динамических систем// Сб. мат. XXVI Междунар. конф. «Математика. Компьютер. Образование» (Пущинский центр РАН, 28 января02 февраля 2019 г.).

8. Андреева И. А., Андреев А. Ф. Фазовые портреты одного семейства кубических систем в круге Пуанкаре. - Saarbrucken: Lambert, 2017.

9. Андреева И. А., Андреев А. Ф. Фазовые портреты одного семейства кубических систем в круге Пуанкаре, I// Вестн. РАЕН. - 2017. - 4. - С. 8-18.

10. Андреева И. А., Андреев А. Ф. Фазовые портреты одного семейства кубических систем в круге Пуанкаpe// XXVIII Крымская осенняя математическая школа-симпозиум по спектральным и эволюционным задачам (КРОМШ-2017), 2017. - С. 53-55.

11. Андреева И. А., Андреев А. Ф. Результаты исследования семейства кубических динамических систем в круге Пуанкаре// Тез. докл. междунар. конф. «Геометрические методы в теории управления и математической физике» (Рязань, 25-28 сентября 2018 г.). - С. 6.

12. Андреева И. А., Андреев А. Ф. Фазовые портреты семейства кубических систем в круге Пуанкаре, II// Вестн. РАЕН. - 2018. - 4. - С. 11-15.

13. Андреева И. А., Андреев А. Ф. О картине траекторий некоторого семейства кубических динамических систем// Сб. мат. междунар. конф. «XXIX Крымская осенняя математическая школа-симпозиум по спектральным и эволюционным задачам», 2018. - С. 99-101.

14. Андреева И. А., Андреев А. Ф. Фазовые портреты некоторого семейства кубических динамических систем в круге Пуанкаре, III// Вестн. РАЕН. - 2019. - 2. - С. 20-24.

15. Андреева И. А., Андреев А. Ф., Садовский А. П., Детченя Л. В., Маковецкая Т. В. Нильпотентные центры кубических систем// Диффер. уравн. - 2017. - 53, № 8. - С. 1003--1008.

16. Андронов А. А., Леонтович Е. А., Гордон И. И., Майер А. Г. Качественная теория динамических систем второго порядка. - М.: Наука, 1966.

17. Пуанкаре А. О кривых, определяемых дифференциальными уравнениями. - М.: ОГИЗ, 1947.

18. Andreeva I., Andreev A. Investigation of a family of cubic dynamic systems// Vibroeng. Proc. — 2017. 15. - P. 88-93.

19. Andreeva I. A., Andreev A. F. On a behavior of trajectories of a certain family of cubic dynamic systems in a Poincaré circle// J. Phys. Conf. Ser. — 2018. - 1141. — 012114.

20. Andreeva I., Andreev A. Phase portraits of cubic dynamic systems in a Poincaré circle// in: Differential Equations. Theory and Current Research (Moschandreou T. E., eds.). — London, UK, 2018.

21. Andreeva I. A., Andreev A. F., Detchenya L. V., Makovetskaya T. V., Sadovskii A. P. Nilpotent centers of cubic systems// Differ. Equations. — 2017. — 53, № 8. - P. 975-980. 
22. Andreeva I. A., Efimova T. O. Phase portraits of a special class of dynamic systems in a Poincaré circle// J. Phys. Conf. Ser. - 2019. - 1236. - 012053.

23. Poincaré H. Les methodes nouvelles de la mecanique celeste. - Paris, 1892-99.

Андреева Ирина Алексеевна

Санкт-Петербургский политехнический университет Петра Великого

E-mail: irandr@inbox.ru

Андреев Алексей Федорович

Санкт-Петербургский государственный университет

E-mail: irandr@inbox.ru 